\title{
Winners and losers from the gradual formation of trading blocs*
}

\author{
Inés Macho-Stadler ${ }^{\dagger}$ and Licun Xue
}

First version: May, 2004, this version: February 2006

\begin{abstract}
Although global free trade is efficient, each country's benefit from free trade depends on the path that leads to the global free trade agreement. Using a dynamic model of trading bloc formation, we show that when global free trade is reached gradually, the countries that are initially excluded gain less than the rest and may be even made worse-off by the final free trade agreement, compared with the initial state of no trading blocs.
\end{abstract}

\section{Introduction}

Does free trade benefit all? Ohyama [9], Kemp and Wan [3] show that it is possible that no country suffers from the formation of a trading bloc and Konishi, Kowalczyk and Sjöström[5] show that it is possible that no country suffers from global free trade. We revisit the issue whether free trade

*We would like to thank an anonymous referee for his/her detailed and constructive comments. We would also like to thank Effrosyni Diamantoudi and David Pérez-Castrillo for helpful remarks and criticisms. The previous version of the paper has circulated under the title: "Does free trade benefit all?". The first author gratefully acknowledges the financial support from projects BEC2003-01132, 2001SGR-00162 and Barcelona EconomicsCREA. The second author would like to thank SSHRC and FQRSC for financial support.

${ }^{\dagger}$ Department of Economics and CODE, Fac CCEE, Universitat Autònoma de Barcelona, Edifici B, E-08193 Bellaterra (Barcelona), Spain.

${ }^{\ddagger}$ CIREQ, Montreal and Department of Economics, McGill University, mailing address: 855 Sherbrooke Street West, Montreal, Quebec, Canada H3A 2 T7. 
benefits all in a dynamic strategic model where countries can form trading blocs endogenously. By forming a trading bloc, member countries agree on the tariffs on mutual trade (often implying free trade among members, i.e., the emergence of customs union) and on the tariffs on their imports from non-members. In our model, although global free trade eventually emerges as the equilibrium outcome, some countries may benefit more than others do. In fact, it is possible for a country to be worse off compared with the initial situation where no trading bloc is formed (i.e., the noncooperative Nash equilibrium situation). The driving forces of such a possibility are the negative externalities a trading bloc imposes on outsiders and the strategic behavior the countries entertain in forming a trading bloc. If those externalities are very high, the countries who are left out of the intermediate trading bloc obtain a lower welfare in the eventual free trade than the initial situation.

The literature on international trade agreements often considers a twostages process in order to depict the timing of such relationships. In the first stage, which is often referred to as the trade union (coalition) formation stage, countries choose their partners in the trade agreements. In the second stage, each trade union sets tariffs given the partition from the first stage. In this stage, countries within each trade union behave cooperatively to maximize their joint welfare, while the interactions among different trade unions are noncooperative.

Sharing this common structure, models in the literature differ in the formalization of both stages. The differences in the second stage depend on the underlying economic model, while the differences in the first stage depend on the approach to the coalition formation procedure. All the papers share the common property that the grand coalition is efficient, which is often equivalent to asserting that free trade is the efficient organization.

Concerning the first stage, the core has been considered as a natural solution concept for analyzing a word-wide trade agreement and free trade. For example, Riezman [10] and Macho-Stadler, Pérez-Castrillo and Ponsatí [8] use the core to identify the stable partition of countries into customs unions. These papers rule out the possibility of international transfers but account for the externalities that a customs union inflicts on other countries. Kowalczyk and Sjöström [6] and Konishi et al. [5] also take a cooperative approach to the first stage of the game, but they allow for monetary transfers among countries. However, these models, like Ohyama [9] and Kemp and Wan [3], do not consider externalities among the coalitions of countries, which simplifies the analysis of the transfer scheme within the countries when they sign a 
trade agreement. Kowalczyk and Sjöström [6], in a many-country monopoly trade model, show that the grand coalition may require international income transfers. They derive a formula for the transfers that leads to free trade (the grand coalition forms) and supports the Shapley value as a core allocation. Konishi et al. [5] show that global free trade can be an immediate outcome without any customs union ever forming provided that transfer payments are possible and customs unions have no effects on non-member countries.

Burbidge, DePater, Myers and Sengupta [2] consider a one-shot noncooperative game of coalition formation in the first stage where countries simultaneously announce their partners for trading blocs. Their model allows for both transfers and externalities. They show that when there are more than two countries, global free trade may not be an equilibrium outcome. Our paper complements the previous papers by considering a dynamic noncooperative model of trading blocs formation. With such a framework, a subset of countries forming a customs union or a trading bloc does not preclude the global free trade agreement from being reached eventually. Casual observation does support gradual formation of trade unions. For example, before NAFTA was formed, the United States and Canada were already enjoying their bilateral trade agreements. Similarly, the European Union started with only six countries, to reach the present membership through gradual admittance of new members.

Concerning the second stage, different models of custom unions have been analyzed in the literature. Kennan and Riezman [4] construct a pure exchange economy in which commodity demands in each country are generated by a linear demand system. In their model all countries charge optimal tariffs given the structure of customs union and the tariffs charged by other countries, but international transfers are not allowed. As the authors point out, the analysis of optimal tariffs is very complicated even when trade-agreements are not considered. They generate some examples with three countries and three goods that highlight some interesting aspects of the problem. In particular, the formation of custom unions can improve its members welfare relative to free-trade. Burbidge et al. [2] consider a one-good model of capital tax competition with interstate trade of mobile capital for the consumption good. While their model is quite appealing, it is not analytically tractable. They provide examples to illustrate, for example, that the grand coalition may not be an equilibrium outcome. In view of this, we employ a very simple three country model as in Macho-Stadler et al. [8] that is analytically solv- 
able even for asymmetric situations ${ }^{1}$ and at the same time generate payoff configurations qualitatively similar to those in Kennan and Riezman [4] and Burbidge et al. [2]. Krishna [7] uses a model of imperfect competition similar to ours and examine how bilateral trade agreement affects multilateral trade liberalization.

In a recent paper, Aghion, Antràs, and Helpman [1] consider a threecountry dynamic bargaining game where one country plays the role of a "leader" or agenda setter who has the power to choose how negotiation is to be conducted (multilaterally or sequentially). Their second-stage game is a partition function game. They analyze the incentive of the agenda setter in choosing the form of negotiation and show that free trade emerges when payoffs exhibit grand-coalition superadditivity.

We show that in equilibrium of our model the grand coalition is always formed and engages in free trade. However, the grand coalition is not necessarily formed in one step. Indeed, if countries are patient, a two country trading bloc is formed first, after which it merges with the third country to form the grand coalition. In doing so, the two countries that form the initial trading bloc extract more surplus at the expense of the third country's welfare in the final free trade agreement. In fact, the third country may be worse off in the end compared with the initial position where no trading bloc is formed.

The organization of the paper is as follows. Section 2 presents the model. Section 3 examines the welfare properties of different trading bloc structures and determines each country's payoff as a function of the current trade bloc structure and the sequence that leads to this trade bloc structure. Section 4 presents and analyzes the dynamic game of trading bloc formation. Proofs are relegated to the Appendix.

\section{The model}

Consider a three country model where a single homogeneous good is produced and sold in each period. Countries are indexed by 1, 2, and 3. Each country has one firm (also indexed by 1,2, and 3) that produces the good and sells it in the domestic and foreign markets. The markets in the different countries are assumed to be segmented. The inverse demand function of country $i$, for

\footnotetext{
${ }^{1} \mathrm{Yi}[11]$ also provides a solvable model but only for symmetric countries.
} 
$i=1,2,3$, is

$$
p_{i}=a_{i}-Q_{i},
$$

where $p_{i}$ is the domestic price and $Q_{i}$ is the total amount sold in country $i$. Let $q_{i j}$ denote the quantity sold in country $i$ by firm $j$. Then,

$$
Q_{i}=q_{i 1}+q_{i 2}+q_{i 3}
$$

As in Krishna [7], it is assumed that there is a competitively produced numeraire good and that this numeraire good is freely traded across countries to settle the balance of trade. ${ }^{2}$

Country $i$ sets non-negative tariffs $t_{i j} \geq 0$ on firm $j$ 's product sold in country $i$, with $t_{i i}$ interpreted as quantity tax on the domestic firm ${ }^{3}$. The production cost function of firm $i$ is $C_{i}(q)=c_{i} q_{i}$, where $0 \leq c_{1} \leq c_{2} \leq c_{3}$ and $q_{i}$ is the quantity produced by firm $i$. In each country $i$, firms choose quantity in a noncooperative fashion given the tariffs $\left(t_{i 1}, t_{i 2}, t_{i 3}\right)$. The effective unit costs of firm $j$ 's product sold in country $i$ are $\left(c_{i}+t_{i j}\right)$ if the solution leads firm $j$ to produce in equilibrium. The reason for choosing such a model is two-fold: it is analytically tractable and can generate payoff structures similar to those in the literature. Moreover, for our purpose, a three country model is sufficient.

If the solution is interior, in the unique Cournot (Nash) equilibrium, firm $j$ sells the following quantity in country $i$ :

$$
q_{i j}=\frac{a_{i}+\left(c_{\ell}+t_{i \ell}\right)+\left(c_{k}+t_{i k}\right)-3\left(c_{j}+t_{i j}\right)}{4},
$$

where $j, k, l \in\{1,2,3\}$ are distinct numbers. In equilibrium, the output that firm $j$ sells in country $i$ is decreasing in its own effective costs and increasing in its rivals' effective costs. Note that by setting $t_{i j}$ high enough, country $i$ could induce firm $j$ not to sell in $i$ 's domestic market. For simplicity, we assume throughout the paper that the demand in every country is high enough relative to costs, so that in equilibrium all firms are always active in all three markets. ${ }^{4}$ Let $\pi_{i j}$ be firm $j$ 's profits in country $i$. Then,

$$
\pi_{i j}=q_{i j}^{2}
$$

\footnotetext{
${ }^{2}$ This is a two-good model of trade, where a quasilinear utility function generates the demand for the oligopolistic good.

${ }^{3}$ We rule out export subsidies and firm subsidies by assumption.

${ }^{4}$ For asymmetric firms, with $c_{1} \leq c_{2} \leq c_{3}$, a sufficient condition is $a_{i}>11 c_{3}-5 c_{2}-5 c_{1}$. For symmetric firms (equal costs) this reduces to assume that $a_{i}>c$ for every country $i$.
} 
The consumer surplus in country $i$, if all firms sell in this country, is

$$
C S_{i}=\frac{1}{32}\left(3 a_{i}-\sum_{j=1}^{3}\left(c_{j}+t_{i j}\right)\right)^{2}
$$

Note that each firm's profits earned in country $i$ and contribution to consumer welfare in country $i$ depend only on the tariff structure set in country $i$. The domestic firm's profits are increasing in the tariffs applied to foreign firms and decreasing in the tax on its own product. Consumer surplus in each country is increasing in the total quantity sold in that country. This implies that it is decreasing in the effective costs of the firms that sell in the domestic market and hence in the tariffs $t_{i 1}, t_{i 2}$ and $t_{i 3}$.

Total welfare per period in country $i$ is the sum of its consumer surplus, the total profits of the domestic firm, and the total revenue from tariffs/tax:

$$
W_{i}=C S_{i}+\sum_{j=1}^{3} \pi_{j i}+\sum_{j=1}^{3} t_{i j} q_{i j} .
$$

Note that total welfare in country $i$ depends on the whole set of tariffs since the profits of the domestic firm depend on the tariffs set by the three countries.

A group of countries can form a trading bloc. A partition describes how the three countries organize themselves into trading blocs. The set of possible partitions is given by $\mathcal{P}=\{I,[12],[13],[23], N\}$, where,

$$
\begin{aligned}
I & =(\{1\},\{2\},\{3\}) \\
{[i j] } & =(\{i, j\},\{k\}), \text { where } i, j, k \in\{1,2,3\} \text { are distinct } \\
N & =(\{1,2,3\}) .
\end{aligned}
$$

In these partitions, a country can either be a member of a trading bloc or be alone in that it does not belong to any trading bloc and therefore it sets tariffs unilaterally. In the first partition, all three countries are "alone", in which case, they behave noncooperatively by setting their Cournot-Nash tariffs. In the last partition, all three countries form a single trading bloc (the grand coalition) and maximize their joint welfare. Each of the other partitions involves a two-country trading bloc and one country being alone. Given a partition of this type, the trading bloc's objective is to maximize the joint welfare of member countries while the interaction between the trading 
bloc and the third country is noncooperative. That is, we assume that only transfers among countries in the same trading bloc are possible.

For any partition of the countries into trading blocs, we can determine the equilibrium tariffs and taxes given this partition. Taxes on domestic firm decrease both domestic consumer surplus (3) and domestic firm's profits due to the decrease in its production (1) but increase tax revenue. Tariffs on foreign firms decrease domestic consumer surplus (3) while increasing domestic firm's profits and tariff revenue. We show in Appendix 1 that in equilibrium, any country sets zero taxes on the domestic firms, and any trading bloc chooses free trade among its members. In addition, the tariffs on the outsider(s) are increasing in the domestic demand and decreasing in the production costs of the outsider(s). Full characterization of the equilibrium taxes and tariffs is presented in Appendix $1 .^{5}$

Given the above equilibrium taxes and tariffs we can determine each trading bloc's welfare for every partition. Let $W_{i}^{I}$ be country $i$ 's welfare in equilibrium when no trading blocs are formed. Let $W_{T}^{N}$ denote the total (global) welfare when the grand coalition/trading bloc forms. Lastly, given partition $[i j]$, let $W_{i j}^{[i j]}$ be the joint welfare of countries $i$ and $j$ and $W_{k}^{[i j]}$ country $k$ 's welfare.

\section{Payoffs}

In this section we ascertain countries's payoffs associated with any trade agreement structure $P \in\{I,[12],[13],[23], N\}$. We first examine the properties of the welfare functions defined in the previous section.

\subsection{Welfare Properties}

Conditional on ruling out subsidies, the grand coalition yields the highest total welfare (since a tariff agreement for any trading bloc can be mimicked by the grand coalition). As for any trade agreement structure in which two countries form a trading bloc, the next two propositions state the impact of

\footnotetext{
${ }^{5}$ Let us note that autarchy is always a possible outcome, if a country sets tariffs in such a way that no foreign firm sells in the domestic market. However, under our condition on demands, as it is shown in Appendix 1, this does not arise in equilibrium.
} 
this trading bloc on the outsider and the incentives of the two insiders in forming the trading bloc.

Proposition 1 The game is of negative externalities in that when two countries merge the third one suffers: $W_{k}^{[i j]}<W_{k}^{I}$ for any $i, j, k$ distinct.

Therefore, when two countries $i$ and $j$ form a trade union, the third country $k$ 's welfare is reduced, as compared to the situation where no trading bloc is formed and this is true irrespective of the level of demand or production cost in country $k$.

It can be shown that $\left(W_{k}^{[i j]}-W_{k}^{I}\right)$ is decreasing in $a_{i}$ and $a_{j}$ but does not depend on $a_{k}$. In addition, $\left(W_{k}^{[i j]}-W_{k}^{I}\right)$ is increasing in $c_{k}$ and, if demands $a_{i}$ and $a_{j}$ are not too different, it is decreasing in $c_{i}$ and $c_{j}$. Hence, the higher the demands of the two countries in the trading bloc and the more efficient the outsider, the more harmful the agreement is for the outsider.

Proposition 2 Any two countries have an incentive to cooperate. That is, $W_{i j}^{[i j\rfloor}>W_{i}^{I}+W_{j}^{I}$ for $i, j \in\{1,2,3\}$.

It is worth noting that the incentive for two countries to cooperate, measured by $\left(W_{i j}^{[i j]}-W_{i}^{I}-W_{j}^{I}\right)$, increases with the size of their demands, $a_{i}$ and $a_{j}$, and does not depend on the demand of the outsider $a_{k}$. This expression is increasing in the cost of the outsider, $c_{k}$ and, when demands of the two countries are not too different, it is decreasing in the costs of the cooperating countries, $c_{i}$ and $c_{j}$. Hence, the higher the demands of the two firms entering an agreement and the less efficient the outsider, the more incremental surplus two countries will generate by forming a trading bloc.

\subsection{Payoffs Associated with Each Partition}

When a new trading bloc is created, the change in each member country's welfare depends on the surplus generated by the new trade agreement and the sharing rule the trading bloc adopts. To characterize each country's payoff, we assume that members of the new trading bloc share equally the incremental surplus ${ }^{6}$ (possibly via transfers). In doing so, we take the view

\footnotetext{
${ }^{6}$ Different sharing rules can be employed without altering the qualitative results, as we shall illustrate in the next subsection.
} 
that each country's payoff depends not only on the current trade agreement structure but also on the sequence of trade agreement structures that precede the current one. In particular, how the three countries in our framework share the gain from free trade when the grand coalition forms depends on whether the grand trading bloc forms directly or through some intermediate stage where two of the countries form a trading bloc first.

If all countries are alone, each country $i$ 's, where $i \in\{1,2,3\}$, status quo payoff is $W_{i}^{I}$. If no trading bloc emerges, country $i$ 's payoff in each period remains $W_{i}^{I}$. A subset of countries can form a trading bloc. If the first trading bloc has only two members, then they share the surplus equally while the third country sees its welfare reduced. Each country's payoff remains the same until the first trading bloc merges ${ }^{7}$ with the third country to form the grand coalition, in which case all countries share the incremental surplus equally. Another possibility is that the three countries decide to form the grand coalition directly. Once the grand coalition forms, each country's payoff in each period stays the same thereafter. Therefore, each country's payoff only depends on the sequence of distinct partitions that have emerged thus far. The set of possible sequences of partitions, each of which starts with $I$, is

$$
\mathcal{S}=\{I, I-[i j], I-[i j]-N, I-N\}_{i, j \in N, i \neq j},
$$

where $I$ depicts, for example, that no trading bloc has been formed and $I$ remains the current partition, while $I-[i j]-N$ depicts that the grand coalition forms via intermediate partition $[i j]$.

We now start with $I$ and determine recursively the payoff allocations associated with each of the above sequences. We shall denote by $V_{i}(S)$ the payoff (per period) of country $i$ following sequence $S \in \mathcal{S}$. Obviously, $V_{i}(I)=W_{i}^{I}$.

If the grand coalition is formed in one step, as denoted by sequence $I-N$, the incremental surplus is

$$
\Delta(I-N)=W_{T}^{N}-\left(W_{1}^{I}+W_{2}^{I}+W_{3}^{I}\right) .
$$

In this case country $i$ receives the payoff

$$
V_{i}(I-N)=W_{i}^{I}+\frac{1}{3} \Delta(I-N) .
$$

\footnotetext{
${ }^{7}$ We assume that a trading bloc never dissolves but can merge with the third country.
} 
If trading bloc $\{i, j\}$ is formed (from $I$ ), it generates a surplus in the amount of

$$
\Delta(I-[i j])=W_{i j}^{[i j]}-\left(W_{i}^{I}+W_{j}^{I}\right) .
$$

The payoff of country $\ell \in\{i, j\}$ associated with sequence $I-[i j]$ is

$$
V_{\ell}(I-[i j])=W_{\ell}^{I}+\frac{1}{2} \Delta(I-[i j]),
$$

while country $k$ 's (who stays isolated) payoff is

$$
V_{k}(I-[i j])=W_{k}^{[i j]} .
$$

Consider now the case in which the grand coalition is formed through an intermediary step where two countries, $i$ and $j$, form a trade union first. This corresponds to the sequence $(I-[i j]-N)$.

The incremental surplus generated by forming the grand coalition via an intermediary trading bloc $\{i, j\}$ is

$$
\Delta(I-[i j]-N)=\left(W_{T}^{N}-\left(W_{i j}^{[i j]}+W_{k}^{[i j]}\right)\right) .
$$

Countries' payoffs associated with the sequence $(I-[i j]-N)$ are as follows:

$$
\begin{aligned}
V_{\ell}(I-[i j]-N) & =V_{\ell}(I-[i j])+\frac{1}{3} \Delta(I-[i j]-N) \text { for all } \ell \in\{i, j\}, \\
V_{k}(I-[i j]-N) & =V_{k}(I-[i j])+\frac{1}{3} \Delta(I-[i j]-N) .
\end{aligned}
$$

Once we have determined each country's per period payoff associated with every sequence in $\mathcal{S}$, we proceed to present some properties of the countries' payoff functions.

Proposition 3 If the grand coalition eventually forms, being left out in the first round always results in the worst final payoff. Formally,

$$
V_{k}(I-[i j]-N)<\min \left\{V_{k}(I-[j k]-N), V_{k}(I-[i k]-N), V_{k}(I-N)\right\}
$$

for distinct $i, j$, and $k$.

The next proposition shows that among the sequences leading to the (eventual) formation of the grand coalition, any two countries prefer the one in which they form a trading bloc first. 
Proposition 4 Any pair of countries $i$ and $j$ benefit by forming a trading bloc first. Formally,

$$
V_{i}(I-[i j]-N)>\max \left\{V_{i}(I-[j k]-N), V_{i}(I-N)\right\}
$$

for distinct $i, j$, and $k$.

Recall that when countries $i$ and $j$ form a trading bloc first, a negative externality is imposed on country $k$. In fact, such a negative externality may be large enough to make country $k$ worse off in the grand trading bloc than when all countries are independent, although once $i$ and $j$ form a trading bloc, it is in $k$ 's best interest to join them subsequently.

To illustrate the previous results, take the example where countries 1 and 2 are identical with $a_{1}=a_{2}=100$ and $c_{1}=c_{2}=0$, and country 3 has $a_{3}=22$ and $c_{3}=2$. Then the payoff of the countries as a function of the coalition structure and the path are: ${ }^{8}$

\begin{tabular}{|c|c|c|c|}
\hline Sequence $S$ & $V_{1}(S)$ & $V_{2}(S)$ & $V_{3}(S)$ \\
\hline$I$ & 4090.75 & 4090.75 & 335.22 \\
\hline$I-[12]$ & 4471.945 & 4471.945 & 206.852 \\
\hline$I-[12]-N$ & $\mathbf{4 5 7 8 . 9 4 7}$ & $\mathbf{4 5 7 8 . 9 4 7}$ & $\mathbf{3 1 3 . 8 5 4}$ \\
\hline$I-[13]$ & 4266.543 & 4015.703 & 511,013 \\
\hline$I-[13]-N$ & 4492.706 & 4241.866 & 737.176 \\
\hline$I-N$ & $\mathbf{4 4 0 9 . 0 9 3}$ & $\mathbf{4 4 0 9 . 0 9 3}$ & $\mathbf{6 5 3 . 5 6 3}$ \\
\hline
\end{tabular}

In the table it is easy to see that when free trade is reached after some countries have from a trading bloc, the countries involved in a intermediate trading bloc benefit more (and the country left out less) than when free trade is reached in one step. The previous example, also illustrates the possibility that the country left out of the trading bloc may end up worse off at the end than at the initial situation. This is the case for country 3, which is worse off in the end when the grand coalition is formed via $(I-[12]-N)$ than in the singleton case. ${ }^{9}$

While the above properties that our payoff functions exhibit (Propositions 1,2 and 3) can be attributed to the Cournot model we employ, other models

\footnotetext{
${ }^{8}$ Note that $(I-[23]-N)$ will be similar to $(I-[13]-N)$ changing the payoffs of player 1 and 2 since these countries are identical.

${ }^{9}$ It is not the case for country 2 , which is better off if the grand coalition is formed via $(I-[13]-N)$ than in situation $I$.
} 
in the literature share the same characteristics as the previous example. This is the case for the four examples in Kennan and Riezman [4] (pages 77 and 78). Taking the first example of their paper (where countries are symmetric), and adding transfers by applying the equal sharing of the surplus, we can compute the countries' payoffs for the different sequences:

\begin{tabular}{|c|c|c|c|}
\hline Sequence $S$ & $V_{1}(S)$ & $V_{2}(S)$ & $V_{3}(S)$ \\
\hline$I$ & 79.77 & 79.77 & 79.77 \\
\hline$I-[12]$ & 88.56 & 88.56 & 68.80 \\
\hline$I-[12]-N$ & $\mathbf{9 6 . 7 3}$ & $\mathbf{9 6 . 7 3}$ & $\mathbf{7 6 . 9 6}$ \\
\hline$I-N$ & $\mathbf{9 0 . 1 4}$ & $\mathbf{9 0 . 1 4}$ & $\mathbf{9 0 . 1 4}$ \\
\hline
\end{tabular}

Note that as in this example, by merging sequentially, players 1 and 2 may increase their payoff; however, country 3 losses at the end. The same happens in the other examples presented in Kennan and Riezman [4].

The same features are present in the example presented by Burbidge et al. [2]. For their example all countries share the same production technology, a Cobb-Douglas function that uses capital and labour as inputs. Countries differ in their input endowments. Countries 1 and 2 are similar and are relatively capital-abundant. Country 3 has no capital, but has a large labour endowment. Table 1 of their paper summarizes the payoffs in terms of the Nash Equilibrium in capital tax competition as a function of the coalition structure. We add the payoff of the grand coalition when it is reached through the path $(I-[12]-N)$ or $(I-[13]-N)$ and we apply the equal sharing of surplus generated when a trade bloc is formed. Then we have:

\begin{tabular}{|c|c|c|c|}
\hline Sequence $S$ & $V_{1}(S)$ & $V_{2}(S)$ & $V_{3}(S)$ \\
\hline$I$ & 0.0736 & 0.0736 & 0.8235 \\
\hline$I-[12]$ & 0.1217 & 0.1217 & 0.6835 \\
\hline$I-[12]-N$ & $\mathbf{0 . 1 4 6 0}$ & $\mathbf{0 . 1 4 6 0}$ & $\mathbf{0 . 7 0 7 9}$ \\
\hline$I-[13]$ & 0.0771 & 0.0793 & 0.8270 \\
\hline$I-[13]-N$ & 0.0775 & 0.0846 & 0.8327 \\
\hline$I-N$ & $\mathbf{0 . 0 8 3 4}$ & $\mathbf{0 . 0 8 3 4}$ & $\mathbf{0 . 8 3 3 2}$ \\
\hline
\end{tabular}

Note that 1 and 2 receive the highest payoffs by forming a trading bloc first and then subsequently merging with 3 . On the other hand, 3 is worse off compared to the situation with no trading blocs. 


\section{Dynamic Formation of Trading Blocs}

The previous section characterizes the payoffs as a function of the trade agreement structure reached by the countries. In this section we shall examine whether and how the grand trading bloc forms. In particular, we shall identify which of the sequences specified in the previous section emerges as an equilibrium outcome of a dynamic coalition formation game.

The formation of trading blocs is modeled as an infinite horizon dynamic game. For simplicity, all the countries are assumed to have the same discount factor $\delta \in[0,1)$.

Each period $\tau$ consists of two stages. Stage 1 determines the formation of a trading bloc. At stage 2 countries simultaneously set tariffs and firms produce and sell the output in the three markets. Stage 2 determines the payoffs of the three countries as specified in Section 3: the payoffs depend on the current partition of countries and on the sequence of the trading blocs that have been formed previously. The surplus generated by a trading bloc is shared equally among its members ${ }^{10}$.

We consider a sequential bloc formation game with a fixed protocol. In particular, we assume that countries take their actions in stage 1 according to the following exogenously given order $(i, j, k)$. If the grand coalition forms, the game ends. If a two-country trading bloc forms, it behaves like a single entity. We can specify the protocol in such a way that the two-country trading bloc and the third country take actions alternately with the third country acting first. It is worth noting that the order specified here does not affect the equilibrium outcome.

At each period a country or a two-country trade bloc becomes the proposer and makes an offer to form a trading bloc. The game starts with all countries being alone. Country $i$ proposes a trading bloc that includes $i$. All other members of the proposed bloc answer sequentially according to the protocol by saying "yes" or "no". If all members say yes, the bloc forms. Otherwise, $j$ becomes the next proposer. In the next period the protocol selects a country or a trading bloc in the current partition to propose unless the grand coalition has already formed, in which case the game ends.

Formally, at $\tau=1$ :

1.1 Country $i$, selected by the protocol, makes an offer to a subset $B_{1} \subset$ $\{1,2,3\}, i \in B_{1}$, to form a trading bloc. The members of $B_{1} \backslash\{i\}$

\footnotetext{
${ }^{10}$ Note that the sharing rule is exogenously given.
} 
sequentially (following the protocol) decide whether to join or not. The trading bloc $B_{1}$ is formed if all the members agree. If $B_{1}$ contains any country other than $\{i\}$, the sequence is then $S_{1}=I-B_{1}$. Otherwise, no new trading bloc is formed and the sequence is $S_{1}=I$. Let us denote by $P_{1} \in\{I,[12],[13],[23], N\}$ the resulting partition at the end of $\tau=1$.

1.2 Each country $i \in N$ obtains, at $\tau=1$, payoff $V_{i}\left(S_{1}\right)$.

Consider any time $\tau>1$. Let the partition structure after period $\tau-1$ be $P_{\tau-1}$ and the sequence of (distinct) partitions until this time be $S_{\tau-1}$. If $P_{\tau-1}$ is the grand coalition $N$, then the coalition structure after period $\tau$ is $P_{\tau}=P_{\tau-1}$, and the sequence $S_{\tau}=S_{\tau-1}$. Otherwise:

$\tau .1$ A country or a two-country trading bloc in $P_{\tau-1}$ is selected by the protocol. The proposer makes an offer to a subset $B_{\tau} \subset P_{\tau-1}$ to form a trading bloc. The proposer has to belong to $B_{\tau}$. The members of $B_{\tau}$ sequentially (following the protocol) decide whether to join or not. The trading bloc $B_{\tau}$ is formed if all the members agree.

$\tau .2$ The coalition structure at time $\tau$ is $P_{\tau}$. The sequence of trading blocs is given by $S_{\tau}=S_{\tau-1}$ if $P_{\tau}=P_{\tau-1}$, and $S_{\tau}=S_{\tau-1}-B_{\tau}$ if $P_{\tau} \neq P_{\tau-1}$. Country $i \in N$ obtains the payoff $V_{i}\left(S_{\tau}\right)$ at time $\tau$.

$V_{i}\left(S_{\tau}\right)$ for $S_{\tau} \in\{I, I-[i j], I-[i j]-N, I-N\}_{i, j \in N, i \neq j}$ is the payoff function defined in the previous section: whenever a new trading bloc forms, its members share the surplus equally and if no new trading bloc forms, every country's payoff remains the same. Note that each country's payoff in pe$\operatorname{riod} \tau$ depends only on the sequence of partitions that lead to the current partition. Country $i$ maximizes $\sum_{\tau=1}^{\infty} \delta^{\tau} V_{i}\left(S_{\tau}\right)$.

Note that in the above process of trading bloc formation, a trading bloc, once formed, cannot dissolve but it remains in the negotiation with the possibility of entering a larger trading bloc. A profile of strategies constitutes a subgame perfect equilibrium if its restriction to every subgame induces a Nash equilibrium for that subgame. As in most of the literature on coalition formation, we shall focus on pure strategy Markov Perfect Equilibrium (MPE) in which each proposing country's strategy only depends on the sequence of (distinct) partitions that have been formed thus far and each responding country's strategy depends only on this sequence and the current 
proposal (but neither depends on the period or the details of the past history of the game such as for how many periods a particular partition of countries has been existed).

Given a MPE, let $E V_{i}\left(S_{\tau}\right)$ be the discounted (at the beginning of $\tau+1$ ) payoff of country $i$ in the subgame where the sequence of partitions formed at period $\tau$ is $S_{\tau}$.

Given that the grand coalition remains together once it is formed, it is obvious that for $S_{\tau} \in\{I-[i j]-N, I-N\}$

$$
E V_{i}\left(S_{\tau}\right)=\sum_{\tau^{\prime}=1}^{\infty} \delta^{\tau^{\prime}-1} V_{i}\left(S_{\tau}\right)
$$

In addition, it is easy to see that

$$
E V_{i}(I-[i j]) \leq \sum_{\tau^{\prime}=1}^{\infty} \delta^{\tau^{\prime}-1} V_{i}(I-[i j]-N),
$$

since the grand coalition is efficient. Moreover,

$$
E V_{i}(I) \leq \max _{j \neq i}\left(V_{i}(I-[i j])+\delta E V_{i}(I-[i j])\right),
$$

since $V_{i}(I-N)<V_{i}(I-[i j]-N)$.

Lemma 5 Consider a sequence ending in a partition [12], [13], or [23] and the subgame following this sequence. Then in every MPE any proposer offers to form the grand coalition and all the countries/trading blocs agree on it.

Lemma 5 says that, for any discount rate, if two countries have formed a trading bloc then the grand coalition will form in the next period. It also implies that $E V_{i}\left(S_{\tau}\right)=\frac{\delta}{1-\delta} V_{i}\left(S_{\tau}-N\right)$ for any sequence $S_{\tau} \in\{I-[12], I-$ $[13], I-[23]\}$.

Now let us consider the countries' behavior following sequence $S_{\tau}=I$. We start with the case where countries have a low discount factor.

Proposition 6 If the discount factor $\delta$ is sufficiently low and the countries are not too asymmetric, in the only MPE the grand coalition is formed at period 1.

We now examine under what conditions a two-country trade bloc is formed first. 
Proposition 7 Let $\left[i^{*} j^{*}\right]$ be the solution to

$$
\max _{[i j] \in\{[12],[13],[23]\}}\left[\frac{1}{2} \Delta(I-[i j])+\left(V_{k}(I)-V_{k}(I-[i j])\right)\right] .
$$

When $\delta$ is high enough, countries $i^{*}$ and $j^{*}$ form a trading bloc first in the unique MPE.

For symmetric countries the result in terms of the discount rate can be stated more precisely:

Corollary 8 Assume that the three countries are identical and let

$$
\underline{\delta}=\frac{V_{i}(I-N)-V_{i}(I-[i j])}{V_{i}(I-[i j]-N)-V_{i}(I-[i j])} \in(0,1) .
$$

Then if $\delta<\underline{\delta}$ the grand coalition forms immediately and if $\underline{\delta}<\delta<1$, the grand coalition forms via an intermediary trading bloc.

\section{Conclusion}

In this paper we show that each country's benefit from free trade depends on how free trade is reached. It is reached directly or via an intermediate stage where only a subset of countries form a trading bloc. When two countries form a trading bloc first, these countries impose a negative externality on the welfare of the third country. If the third country merge with them to form the grand coalition and engage free trade, the payoff to the third country is less than if all countries initially go directly to free trade. In fact, the third country's payoff from free trade can be lower than those in the noncooperative equilibrium.

The analysis will lead to similar results if countries form customs unions (that is, decide to pursue free trade internally and apply common external tariffs to non-members). If an intermediate agreement is available, being kept out of a custom union can clearly hurt a country. 


\section{Appendix 1: Equilibrium taxes and tariffs}

Taxes on a domestic firm decrease both domestic consumer surplus (3) and the domestic firm's profits due to the decrease in its production (1) but increase in tariff/tax revenue. Unless the domestic firm is very inefficient relative to the domestic demand, the negative effect on domestic consumer surplus and profits dominates the positive effect on tariff/tax revenue. If demand in every country is high enough this implies that no taxes should be imposed on the domestic firm. Formally:

Remark 9 In any partition of the countries into trading blocs, taxes on the domestic firm are set equal to zero, i.e., $t_{i i}=0, i=1,2,3$.

Proof of Remark 9. We omit the proof since it is included in the proof of the following Lemmas.

Now, let us consider countries' decisions on tariffs. Obviously, these decisions depend on the existing partition of trading blocs.

Lemma 10 The grand coalition chooses free trade and no taxes on domestic firms, i.e., $t_{i j}=0$ for all $i, j=1,2,3$.

Proof of Lemma 10. The grand coalition sets the vector $\left(t_{i j}\right), i, j=$ $1,2,3$, in order to maximize total welfare $\sum_{i=1}^{3} W_{i}$. It is easy to show that

$\frac{\partial\left(\sum_{\ell=1}^{3} W_{\ell}\right)}{\partial t_{i 1}}-\frac{\partial\left(\sum_{\ell=1}^{3} W_{\ell}\right)}{\partial t_{i 2}}=\left(c_{1}-c_{2}\right)$ and $\frac{\partial\left(\sum_{\ell=1}^{3} W_{\ell}\right)}{\partial t_{i 2}}-\frac{\partial\left(\sum_{\ell=1}^{3} W_{\ell}\right)}{\partial t_{i 3}}=\left(c_{2}-c_{3}\right)$.

Thus, given that $c_{1} \leq c_{2} \leq c_{3}$, we have

$$
\frac{\partial\left(\sum_{\ell=1}^{3} W_{\ell}\right)}{\partial t_{i 1}} \leq \frac{\partial\left(\sum_{\ell=1}^{3} W_{\ell}\right)}{\partial t_{i 2}} \leq \frac{\partial\left(\sum_{\ell=1}^{3} W_{\ell}\right)}{\partial t_{i 3}} .
$$

Moreover, when $a_{i}>11 c_{3}-5\left(c_{1}+c_{2}\right)$, then $\frac{\partial\left(\sum_{\ell=1}^{3} W_{\ell}\right)}{\partial t_{i 3}}<0$ and all of these derivatives are negative, implying that the solution will be free trade and zero taxes (Note that by assuming that $t_{i j} \geq 0$, we rule out the possibility of 
subsidization). Hence, if demands are high enough (a sufficient condition is $a_{i}>11 c_{3}-5 c_{2}-5 c_{1}$ for all $\left.i\right)$ the grand coalition sets free trade.

The intuition behind this result is simple. Tariffs set by country $i$ on country $j$ decrease the domestic consumer surplus and firm $j$ 's profits in country $i$ while increase country $i$ 's revenue from tariffs. However, when demands are high enough as compared to production costs, the negative effects dominate the positive ones. For any country, tariffs on the most efficient firm (i.e., the firm with lowest unit cost) are the most harmful for global welfare. When the countries collude on tariffs, they fully internalize the effects of tariffs. Therefore, if countries' demands are sufficiently high, it is optimal to have all the firms producing in the most efficient way (i.e., not increasing the effective costs of any firm in such a way that this firm does not produce for this market).

Lemma 11 When all countries are alone, in equilibrium country $i$ sets $t_{i j}=\frac{3}{10} a_{i}-\frac{1}{10} c_{i}+\frac{3}{20} c_{k}-\frac{7}{20} c_{j}$, where $i, j$, and $k$ are distinct countries.

Proof of Lemma 11. The Nash equilibrium in tariffs is the fixed point of the best reply functions of the three countries. Country $i$ sets $\left(t_{i j}\right)$, $j=1,2,3$, in order to maximize $W_{i}$. The first order conditions of this problem do not depend on the tariffs set by the other two countries, implying that the Nash equilibrium is in fact an equilibrium in dominant strategies. By analyzing the first order conditions, we conclude that there is no interior solution where country $i$ 's three tariffs take positive values. Domestic welfare $W_{i}$ is decreasing in the tariff on the own firm for all the combinations of the other tariffs (that are compatible with non-negative production levels). Hence, $t_{i i}=0$. When $a_{i}$ is high enough (a sufficient condition is $a_{i}>11 c_{3}-$ $\left.5 c_{2}-5 c_{1}\right)$, tariffs on the imports by the foreign firms are interior and are given by $t_{i j}=\frac{3}{10} a_{i}-\frac{1}{10} c_{i}+\frac{3}{20} c_{k}-\frac{7}{20} c_{j}$ for distinct $i, j$, and $k$.

Since the domestic welfare does not take into account the effects of tariffs on foreign firms' profits, the tariffs on these firms are positive. However, under our assumption on demands all the firms are active in the domestic market. Optimal tariffs are increasing in the domestic demand and decreasing in the production cost of the domestic firm. In addition, the foreign firm that has a cost advantage will pay a higher tariff.

Finally, there are three possible cases (partitions) where two countries form a trading bloc that we have to consider: $(\{1,2\},\{3\}),(\{2,3\},\{1\})$ and $(\{1,3\},\{2\})$. Let us consider the general case of $(\{i, j\},\{k\})$. 
Lemma 12 Assume that countries $i$ and $j$ form a trading bloc and $k$ is the outsider. Then in equilibrium countries in the trading bloc set

a) $t_{i i}=t_{j j}=t_{i j}=t_{j i}=0$ (i.e., free trade within the trading bloc and no taxes on domestic firms) and

b) $t_{\ell k}=\frac{5}{19} a_{\ell}+\frac{1}{19}\left(c_{\ell}+c_{m}\right)-\frac{7}{19} c_{k}$ where $\ell, m \in\{i, j\}$ and $\ell \neq m$,

while country $k$ sets

c) $t_{k \ell}=\frac{3}{10} a_{k}-\frac{1}{10} c_{k}-\frac{7}{20} c_{\ell}+\frac{3}{20} c_{m}$, where $\ell, m \in\{i, j\}$ and $\ell \neq m$.

Proof of Lemma 12. Consider first $\left(W_{i}+W_{j}\right)$, the joint welfare for the countries in the trading bloc $\{i, j\}$. It is easy to check that if the demands are high enough, we have $\frac{\partial\left(W_{i}+W_{j}\right)}{\partial t_{\ell m}}<0$ for all $\ell, m \in\{i, j\}$, implying that it is optimal to set free trade and zero taxes in the trading bloc (given that the possibility of subsidies is ruled out). In fact, doing so is a dominant strategy since, since the best response function for the trading bloc does not depend on the outsider's tariffs. Moreover, when the demands are high enough, the trading bloc sets positive tariffs on the outsider $k$ 's products $t_{\ell k}=$ $\frac{5}{19} a_{\ell}+\frac{1}{19}\left(c_{\ell}+c_{m}\right)-\frac{7}{19} c_{k}$ where $\ell, m \in\{i, j\}$ and $\ell \neq m$. The maximization problem of country $k$ (who is not in the trading bloc) resembles the case where all countries are alone: When demands are high, country $k$ imposes zero taxes on the domestic firm and sets positive tariffs on the foreign firms. These tariffs depend on production costs of the foreign firm: a higher tariff is applied to the more efficient firm. These tariffs are such that both foreign firms sell in the domestic market if domestic demand is high enough.

The outsider sets tariffs in the same way as in the case where the other countries do not reach a trade agreement.

\section{Appendix 2}

Proof of Proposition 1. We show algebraically that $W_{k}^{[i j]}<W_{k}^{I}$ :

$W_{k}^{[i j]}-W_{k}^{I}=\frac{1449}{36100} a_{i} c_{k}-\frac{683}{18050} a_{i} c_{i}+\frac{1449}{36100} a_{j} c_{k}+\frac{439}{36100} a_{i} c_{j}+\frac{439}{36100} a_{j} c_{i}-\frac{6057}{72200} c_{k} c_{i}-$ $\frac{683}{18050} a_{j} c_{j}-\frac{6057}{72200} c_{k} c_{j}+\frac{2117}{18050} c_{i} c_{j}-\frac{261}{36100} a_{i}^{2}-\frac{261}{36100} a_{j}^{2}+\frac{3159}{72200} c_{k}^{2}-\frac{557}{144400} c_{i}^{2}-\frac{557}{144400}$ $c_{j}^{2}$

First of all, note that $\left(W_{k}^{[i j]}-W_{k}^{I}\right)$ does not depend on $a_{k}$. In addition, it is decreasing in $a_{j}$ (respectively in $a_{i}$ ) :

$\frac{\partial\left(W_{k}^{[i j]}-W_{k}^{I}\right)}{\partial a_{j}}=\frac{1}{36100}\left(1449 c_{k}+439 c_{i}-1366 c_{j}-522 a_{j}\right)<0$. 
In order to show that $W_{k}^{\{i j\}}-W_{k}^{I}<0$, given that $\frac{\partial\left(W_{k}^{[i j]}-W_{k}^{I}\right)}{\partial a_{j}}<0$, we prove that the inequality holds for the lowest values of $a_{i}$ and $a_{j}$ that are compatible with the assumption that each firm is active in all three markets. Let $a$ be this minimum and set $a_{i}=a_{j}=a$. Then:

$$
\begin{aligned}
& \left(W_{k}^{[i j]}-W_{k}^{I}\right)=\frac{1449}{18050} a c_{k}-\frac{927}{36100} a c_{i}-\frac{927}{36100} a c_{j}-\frac{6057}{72200} c_{k} c_{i}-\frac{6057}{72200} c_{k} c_{j}+ \\
& \frac{2117}{18050} c_{i} c_{j}-\frac{261}{18050} a^{2}+\frac{3159}{72200} c_{k}^{2}-\frac{557}{144400} c_{i}^{2}-\frac{557}{144400} c_{j}^{2} .
\end{aligned}
$$

This expression is increasing in $c_{k}$ and decreasing in $c_{i}$ and $c_{j}$. Hence, we have to verify three different cases. Imagine that $k$ is the most efficient country (country 1). Then, the inequality holds for the case where the three countries have the same cost:

$$
\left(W_{k}^{[i j]}-W_{k}^{I}\right)=-\frac{261}{18050}(c-a)^{2}<0,
$$

then the inequality holds everywhere.

Now consider that $k$ is the less efficient country (country 3). Then if the inequality holds for $c_{k}=\frac{1}{11} a$ and $c_{i}=c_{j}=0$, then the inequality holds for all combinations of parameters in this region. This is the case since at this point:

$$
\left(W_{k}^{[i j]}-W_{k}^{I}\right)=-\frac{59409}{8736200} a^{2}<0 .
$$

Finally, if $k$ is the intermediary country in efficiency terms (country 2), then the inequality holds for $c_{k}=c_{i}=\frac{1}{11} a$ and $c_{j}=0$ :

$$
\left(W_{k}^{[i j]}-W_{k}^{I}\right)=-\frac{172277}{17472400} a^{2}<0,
$$

and hence it holds everywhere.

This proves the result given our assumption on demands.

For completeness let us remark how $\left(W_{k}^{[i j]}-W_{k}^{I}\right)$ changes with costs. This difference is increasing in $c_{k}$ :

$\frac{\partial\left(W_{k}^{[i j]}-W_{k}^{I}\right)}{\partial c_{k}}=\frac{1449}{36100} a_{i}+\frac{1449}{36100} a_{j}-\frac{6057}{72200} c_{i}-\frac{6057}{72200} c_{j}+\frac{3159}{36100} c_{k}>0$,

and decreasing in the cost of the countries in the trading bloc if these countries are not too different in demand:

$\frac{\partial\left(W_{k}^{[i j]}-W_{k}^{I}\right)}{\partial c_{i}}=\frac{1}{72200}\left(-2732 a_{i}+878 a_{j}-6057 c_{k}+8468 c_{j}-557 c_{i}\right)$.

The larger the demand parameter of country $i$ and the smaller the demand parameter of country $j$ the more negative is this derivative. Since the opposite happens for $\frac{\partial\left(W_{k}^{\{i j\}}-W_{k}^{I}\right)}{\partial c_{j}}$ both are negative if $a_{i}$ and $a_{j}$ are not too different.

Proof of Proposition 2. We show algebraically that $W_{i j}^{[i j]}>W_{i}^{I}+W_{j}^{I}$. 
$W_{i j}^{[i j]}-W_{i}^{I}-W_{j}^{I}=\frac{113}{950} a_{j} c_{j}-\frac{439}{1900} a_{i} c_{j}-\frac{439}{1900} a_{j} c_{i}+\frac{71}{1900} a_{i} c_{k}+\frac{113}{950} a_{i} c_{i}+\frac{71}{1900} a_{j} c_{k}-$ $\frac{217}{950} c_{i} c_{j}-\frac{213}{3800} c_{i} c_{k}-\frac{213}{3800} c_{j} c_{k}+\frac{71}{1900} a_{i}^{2}+\frac{71}{1900} a_{j}^{2}+\frac{1507}{7600} c_{i}^{2}+\frac{1507}{7600} c_{j}^{2}+\frac{71}{3800} c_{k}^{2}$.

Note that this expression does not depend on $a_{k}$ and is increasing in $a_{i}$ and $a_{j}$ :

$$
\frac{\partial\left(W_{i j}^{[i j]}-W_{i}^{I}-W_{j}^{I}\right)}{\partial a_{i}}=-\frac{439}{1900} c_{j}+\frac{71}{1900} c_{k}+\frac{113}{950} c_{i}+\frac{71}{950} a_{i}>0 .
$$

Hence, it suffices to show that it is positive for the lowest value of $a_{i}=a_{j}=a$. The expression becomes:

$$
\begin{aligned}
& \quad-\frac{213}{1900} a c_{j}-\frac{213}{1900} a c_{i}+\frac{71}{950} a c_{k}-\frac{217}{950} c_{i} c_{j}-\frac{213}{3800} c_{i} c_{k}-\frac{213}{3800} c_{j} c_{k}+\frac{71}{950} a^{2}+\frac{1507}{7600} c_{i}^{2}+ \\
& \frac{1507}{7600} c_{j}^{2}+\frac{71}{3800} c_{k}^{2} .
\end{aligned}
$$

The expression above is decreasing in $c_{i}$ and $c_{j}$ and increasing in $c_{k}$.

$$
\frac{\partial\left(W_{i j}^{[i j]}-W_{i}^{I}-W_{j}^{I}\right)}{\partial c_{i}}=-\frac{213}{1900} a-\frac{217}{950} c_{j}-\frac{213}{3800} c_{k}+\frac{1507}{3800} c_{i}<0 \text { (and similar with }
$$
respect to $\left.c_{j}\right)$, and

$$
\frac{\partial\left(W_{i j}^{[i j]}-W_{i}^{I}-W_{j}^{I}\right)}{\partial c_{k}}=\frac{71}{950} a-\frac{213}{3800} c_{i}-\frac{213}{3800} c_{j}+\frac{71}{1900} c_{k}>0 .
$$

Since $\left(W_{i j}^{[i j]}-W_{i}^{I}-W_{j}^{I}\right)$ is increasing in $c_{k}$ and decreasing in $c_{i}$ and $c_{j}$ we have to verify three different cases. Imagine that $k$ is the most efficient country (country 1). Then if the inequality holds for the lowest $c_{1}, c_{1}=0$, and for the highest $c_{2}$ and $c_{3}$, a largely sufficient condition is $c_{2}=c_{3}=\frac{1}{11} a$, then the inequality always holds. This is the case since at this point:

$$
\left(W_{i j}^{[i j]}-W_{i}^{I}-W_{j}^{I}\right)=\frac{1349}{24200} a^{2}>0 \text {. }
$$

Now consider that $k$ is the less efficient country (country 3). Then if the inequality holds for $c_{i}=c_{j}=c_{k}=c$ then the inequality holds for all combination of parameters in this region. This is guaranteed because at this point:

$$
\left(W_{i j}^{[i j]}-W_{i}^{I}-W_{j}^{I}\right)=\frac{71}{950}(a-c)^{2}>0 .
$$

Finally, if $k$ is the intermediary country in efficiency terms (country 3), then since the inequality holds for $c_{1}=c_{2}=c$ and $c_{3}=11 a$,

$$
\left(W_{i j}^{[i j]}-W_{i}^{I}-W_{j}^{I}\right)=\frac{1}{7600}\left(172407 a^{2}+1223 c^{2}-24066 a c\right)>0,
$$

then it holds everywhere for this last case.

This proves the result given our assumption on demands.

Proof of Proposition 3. First of all note that

$V_{i}(I-[i m]-N)-V_{i}(I-N)=\frac{1}{6} \Delta(I-[i m])+\frac{1}{3}\left(V_{k}(I)-V_{k}(I-[i m])\right)$,

where the two terms in the right side are positive (by Proposition 1 and 2 ), and forming an intermediary trading bloc is always profitable for the 
countries involved. Hence, $\min \left\{V_{k}(I-[j k]-N), V_{k}(I-[i k]-N), V_{k}(I-\right.$ $N)\}=V_{k}(I-N)$.

We shall show that $V_{k}(I-[i j]-N)-V_{k}(I-N)<0$.

$V_{k}(I-[i j]-N)-V_{k}(I-N)=\frac{24401}{433200} a_{i} c_{j}+\frac{24401}{433200} a_{j} c_{i}+\frac{36521}{433200} a_{i} c_{k}-$ $\frac{40579}{433200} a_{j} c_{j}+\frac{53}{1200} a_{k} c_{i}+\frac{36521}{433200} a_{j} c_{k}+\frac{53}{1200} a_{k} c_{j}+\frac{23663}{86640} c_{i} c_{j}-\frac{67}{1200} a_{k} c_{k}+\frac{6233}{86640} c_{i} c_{k}+$ $\frac{6233}{86640} c_{j} c_{k}-\frac{6781}{288800} a_{i}^{2}-\frac{40579}{433200} a_{i} c_{i}-\frac{6781}{288800} a_{j}^{2}-\frac{13}{800} a_{k}^{2}-\frac{30487}{173280} c_{i}^{2}-\frac{30487}{173280} c_{j}^{2}-\frac{22237}{173280} c_{k}^{2}$.

The expression $\left(V_{k}(I-[i j]-N)-V_{k}(I-N)\right)$ is decreasing in the demand of the three countries.

$$
\begin{aligned}
& \frac{\partial\left(V_{k}(I-[i j]-N)-V_{k}(I-N)\right)}{\partial a_{j}}=\frac{53}{1200} c_{i}+\frac{53}{1200} c_{j}-\frac{67}{1200} c_{k}-\frac{13}{400} a_{k}<0 . \\
& \frac{\partial\left(V_{k}(I-[i j]-N)-V_{k}(I-N)\right)}{\partial a_{i}}=\frac{24401}{433200} c_{j}+\frac{36521}{433200} c_{k}-\frac{6781}{144400} a_{i}-\frac{40579}{433200} c_{i}<0
\end{aligned}
$$

and similarly for the derivative with respect to $a_{j}$ by symmetry. Hence, if $\left(V_{k}((i j), N)-V_{k}(N)\right)$ is negative for the highest demand $a$ for the three countries, it would be negative everywhere. For a common (and high) demand $a$ we can rewrite:

$$
\begin{gathered}
V_{k}(I-[i j]-N)-V_{k}(I-N)=\frac{197}{28880} a c_{i}+\frac{197}{28880} a c_{j}+\frac{3257}{28880} a c_{k}+\frac{23663}{86640} \\
c_{i} c_{j}+\frac{6233}{86640} c_{i} c_{k}+\frac{6233}{86640} c_{j} c_{k k}-\frac{3651}{57760} a^{2}-\frac{30487}{173280} c_{i}^{2}-\frac{30487}{173280} c_{j}^{2}-\frac{22237}{173280} c_{k}^{2} .
\end{gathered}
$$

When $a$ goes to infinity this expression is negative.

Proof of Proposition 4. Similar to the proof of Proposition 3.

Proof of Lemma 5. For all the countries the relevant comparison is not to form the grand coalition and then receive a payoff equal to $V_{i}\left(S_{\tau}\right)+\delta E V_{i}\left(S_{\tau}\right)$ or to propose the grand coalition and agree on forming it in which case the expected payoff is $\sum_{\tau=1}^{\infty} \delta^{\tau-1} V_{i}\left(S_{\tau}-N\right)$ for any $S_{\tau} \in$ $\{I-[12], I-[13], I-[23]\}$. Since $E V_{i}\left(S_{\tau}\right)<\sum_{\tau=1}^{\infty} \delta^{\tau-1} V_{i}\left(S_{\tau}-N\right)$ the lemma holds.

Proof of Proposition 6. The grand coalition is formed in the MPE if the following two conditions hold:

(i) the proposer $i$ prefers the grand coalition to any other outcome:

$\sum_{\tau=1}^{\infty} \delta^{\tau-1} V_{i}(I-N) \geq \max \left\{V_{i}(I)+\delta E V_{i}(I), V_{i}(I-[i j])+\delta E V_{i}(I-[i j])\right.$ for $\left.j \neq i\right\}$

(ii) the other two countries agree:

$$
\sum_{\tau=1}^{\infty} \delta^{\tau-1} V_{j}(I-N) \geq V_{j}(I)+\delta E V_{j}(I)
$$


When $\delta \rightarrow 0$ the countries care only about their current payoffs. The previous conditions can be rewritten as:

$$
V_{i}(I-N) \geq \max \left\{V_{i}(I), V_{i}(I-[i j]) \text { for } j \neq i\right\}
$$

and

$$
V_{j}(I-N) \geq V_{j}(I) \text { for } j \neq i
$$

Since the grand coalition is efficient and the surplus is shared equally, the second inequality holds while the first one holds if the countries are not too asymmetric.

Proof of Proposition 7. When $\delta$ goes to 1 , waiting to be the proposer is costless, the relevant comparison for any country is:

$$
V_{i}(I-[i j]-N) \geq \max \left\{V_{i}(I), V_{i}(I-N)\right\}
$$

for $i, j, k$ distinct.

Since the condition is satisfied for any pair of countries the coalition that would form depends on which one generates more surplus for a two-country trade bloc and which two-country trading bloc implies a higher loss for the third country. ${ }^{11}$ To see this, first note that is easy to see that for the exogenous equal sharing rule of the surplus that we use:

$$
V_{i}(I-[i j]-N)-V_{i}(I-N)=\frac{1}{6} \Delta(I-[i j])+\frac{1}{3}\left(V_{k}(I)-V_{k}(I-[i j])\right) .
$$

Where both terms on the right side are positive. Hence, the coalition that forms is the one that

$$
\underset{[i j] \in\{[12],[13],[23]\}}{\operatorname{Max}}\left[\frac{1}{2} \Delta(I-[i j])+\left(V_{k}(I)-V_{k}(I-[i j])\right)\right]
$$

for $i, j, k$ distinct.

Proof of Corollary 8. For symmetric countries, the grand coalition will always form. The sequence may depend on countries' discount factors. The condition for the grand coalition to form immediately can be written as

$$
\sum_{\tau=1}^{\infty} \delta^{\tau-1} V_{i}(I-N) \geq V_{i}(I-[i j])+\delta \sum_{\tau=1}^{\infty} \delta^{\tau-1} V_{i}(I-[i j]-N) .
$$

\footnotetext{
${ }^{11}$ If only a pair of countries $(i, j)$ satisfies this condition the first time that $i$ or $j$ is called to be the proposer the two-country coalition forms.
} 
From this condition we can determine $\underline{\delta}$ that makes both sides equal. In this case: $\underline{\delta}=\frac{V_{i}(I-N)-V_{i}(I-[i j])}{V_{i}(I-[i j]-N)-V_{i}(I-[i j])}$. Hence, for all $\delta<\underline{\delta}$ the grand coalition forms immediately in the only MPE outcome of the game. Forming a two-country coalition, anticipating that the grand coalition will be formed in the next period, is superior for the proposer than to form the grand coalition straight away if

$$
V_{i}(I-[i j])+\delta \sum_{\tau=1}^{\infty} \delta^{\tau-1} V_{i}(I-[i j]-N) \geq \sum_{\tau=1}^{\infty} \delta^{\tau-1} V_{i}(I-N),
$$

which is equivalent to saying that $\delta>\underline{\delta}$.

\section{References}

[1] Aghion, Philippe, Pol Antràs, and Elhanan Helpman, "Negotiating Free Trade", working paper, 2004.

[2] Burbidge, John B., James A. DePater, Gordon M. Myers and Abhijit Sengupta (1997), "A Coalition-Formation Approach to Equilibrium Federations and Trading Blocs", The American Economic Review 87 (5), 940-956.

[3] Kemp, Murray C., and Henry Y. Wan (1976), "An Elementary Proposition Concerning the Formation of Customs Union," Journal of International Economics 6, 95-97.

[4] Kennan, John and Raymond Riezman (1990), "Optimal Tariffs Equilibria with Custom Unions", The Canadian Journal of Economics 23 (1), 70-83.

[5] Konishi, Hideo, Carsten Kowalczyk and Tomas Sjöström (2003), "Free trade, Customs Unions, and Transfers", mimeo.

[6] Kowalczyk, Carsten and Tomas Sjöström (1994), "Bringing GATT into the Core", Economica 61, 301-367.

[7] Krishna, Pravin (1998), "Regionalism and Multilateralism: A Political Economy Approch", Quarterly Journal of Economics, 113 (1), 227-251. 
[8] Macho-Stadler, Inés, David Pérez-Castrillo and Clara Ponsatí (1998) "Stable Multilateral Trade Agreements", Economica 65, 161-177.

[9] Ohyama, Michihiro (1972), "Trade and Welfare in General Equilibrium," Keio Economic Studies 9, 73-73.

[10] Riezman, Raymond (1985), "Custom Unions and the Core", Journal of International Economics 19 (3/4), 355-365.

[11] Yi, Sang-Seung (2000), "Free-trade Areas and Welfare: An Equilibrium Analysis", Review of International Economics 8 (2), 336-347. 